\title{
Primary Ciliary Dyskinesia that Responded to Long-Term, Low-Dose Clarithromycin
}

\author{
Daisuke Yoshioka $^{1,2}$, Noriho Sakamoto ${ }^{1}$, Yuji Ishimatsu ${ }^{1}$, Tomoyuki Kakugawa ${ }^{1}$, \\ Hiroshi Ishii ${ }^{2}$, Hiroshi Mukae ${ }^{3}$, Jun-ichi Kadota ${ }^{2}$ and Shigeru Kohno ${ }^{1}$
}

\begin{abstract}
A 46-year-old man was referred to our hospital with hemoptysis. He had been diagnosed with chronic sinusitis since childhood, but had received no treatment. Chest CT showed a diffuse centrilobular granular shadow and thickened bronchial walls. Otitis media and decreased spermatic motor ability were identified. In addition, electron microscopy of a biopsy specimen of the nasal mucosa showed a deficiency of inner dynein. Based on these clinical findings, primary ciliary dyskinesia (PCD) was diagnosed and successfully treated with long-term, low-dose clarithromycin. Although the effects of macrolide therapy remain controversial, long-term treatment with low-dose clarithromycin might confer clinical benefits upon patients with PCD.
\end{abstract}

Key words: clarithromycin, immotile cilia syndrome, macrolide, primary ciliary dyskinesia

(Inter Med 49: 1437-1440, 2010)

(DOI: 10.2169/internalmedicine.49.3453)

\section{Introduction}

Primary ciliary dyskinesia (PCD) is a genetic condition associated with abnormal ciliary structure and function that was originally described as immotile cilia syndrome (1). The clinical manifestations of PCD include chronic bronchitis, bronchiectasis, chronic sinusitis, chronic otitis media from infancy and male infertility. Bronchiectasis and pulmonary fibrosis can lead to severe pulmonary dysfunction and death unless excessive and/or abnormal secretions are eliminated (2). However, a standardized treatment for PCD and the means by which to correct the ciliary dysfunction have not been defined, and most recommendations have been extrapolated from those for cystic fibrosis (CF) (3, 4). Here, we describe a patient with PCD in which pulmonary disease was improved by long-term, low-dose treatment with the 14membered macrolide, clarithromycin.

\section{Case Report}

A 46-year-old Japanese man was referred to our hospital for further examination due to hemoptysis. He had been ad- mitted to another hospital 1 month previously with fever and hemoptysis. Antimicrobial therapy and bronchial artery embolization improved these symptoms. He had been diagnosed with chronic sinusitis since childhood, but had received no treatment. A productive cough and occasional hemosputum had persisted since high school. He was not married and had no children.

A physical examination upon admission revealed a body temperature of $37.0^{\circ} \mathrm{C}$, blood pressure of $116 / 72 \mathrm{mmHg}$ and regular pulse of 72 beats/min. Lung auscultation revealed coarse crackles in both lungs. Laboratory findings were:

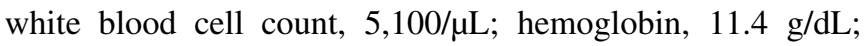
cold agglutinin, $\times 512$; mycoplasmal antibody, below $\times 40$ and immunoglobulin-A, $946 \mathrm{mg} / \mathrm{dL}$. Arterial blood gas analysis while breathing room air showed mild hypoxia and hypercapnia ( $\mathrm{pH}$ 7.406; $\mathrm{PaCO}_{2} 45.2$ Torr; $\mathrm{PaO}_{2} 71.2$ Torr). Sputum microbiology revealed methicillin-sensitive Staphylococcus aureus. Pulmonary function tests demonstrated restrictive (\%VC 63.5\%) and obstructive impairment $\left(\% \mathrm{FEV}_{1}\right.$ $50.0 \%)$. Chest CT showed a diffuse centrilobular granular shadow and thickened bronchial walls (Fig. 1a). Bronchoalveolar lavage fluid contained an increased cell count $(7.7 \times$ $\left.10^{5} / \mathrm{mL}\right)$ with high proportions of neutrophils $(65.8 \%)$ and

${ }^{1}$ The Second Department of Internal Medicine, Nagasaki University School of Medicine, Nagasaki, ${ }^{2}$ Internal Medicine II, Oita University Faculty of Medicine, Oita and ${ }^{3}$ Division of Respiratory Disease, University of Occupational and Environmental Health, Kitakyushu Received for publication January 27, 2010; Accepted for publication April 14, 2010

Correspondence to Dr. Noriho Sakamoto, nsakamot@nagasaki-u.ac.jp 


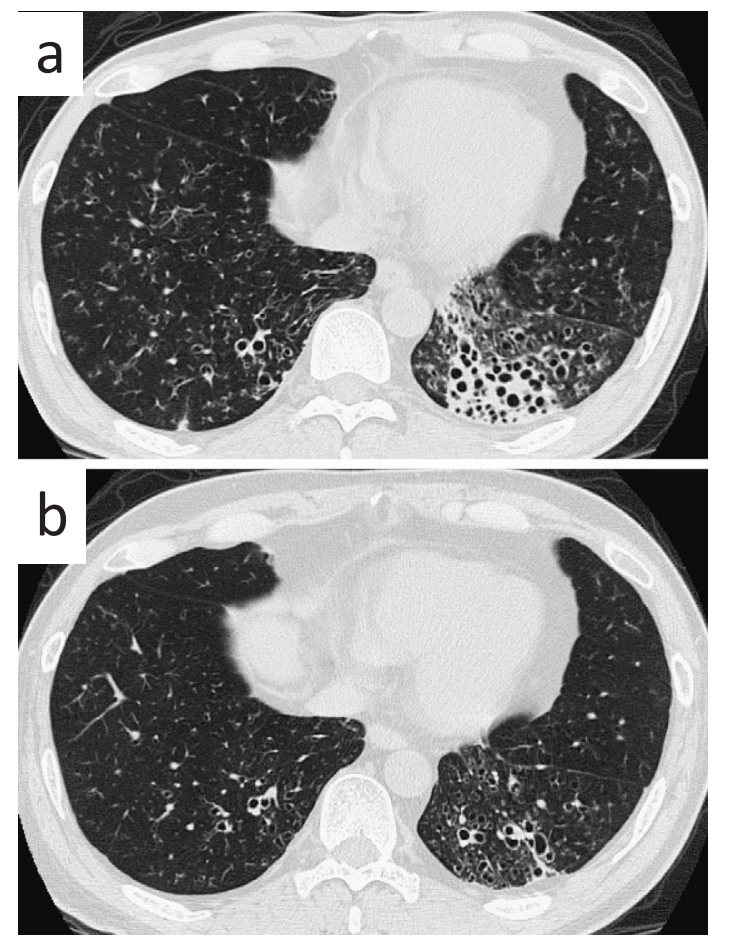

Figure 1. Chest CT scans upon admission and after clarithromycin treatment. Upon admission (a): centrilobular small nodular shadows and bronchiectasis were evident. After two years of treatment (b): centrilobular small nodular shadows had improved.

lymphocytes $(26.7 \%)$ and a low CD4/CD8 ratio of 0.22 . Otitis media with effusion and chronic sinusitis were diagnosed. Spermatic motor ability was also decreased. Electron microscopy of a biopsy specimen from the nasal cavity mucosa revealed a deficiency of inner dynein (Fig. 2). These findings indicated a definitive diagnosis of PCD.

Treatment with clarithromycin (200 mg/day) was started and maintained for two years. No mucoactive drugs or bronchodilators were prescribed. This treatment strategy improved symptoms such as cough and sputum, chest CT findings (Fig. 1b), arterial blood gases $\left(\mathrm{PaO}_{2} 74.3\right.$ Torr) and pulmonary function (\%VC 63.5\%, $\% \mathrm{FEV}_{1} 67.8 \%$; Fig. 3). Symptoms of sinusitis such as nasal obstruction and secretion were also improved.

\section{Discussion}

Lung involvement in PCD is critical because respiratory failure can develop (5). Respiratory management for PCD consists of regular respiratory monitoring, airway clearance by combinations of physiotherapy and physical exercise, and aggressive treatment of upper and lower airway infections (3). Current PCD treatment mainly follows the recommendations for CF. Although drugs such as mucoactive agents, osmolar agents, ion-transport regulators and antibiotics are administered, no empirical evidence supports their effectiveness against $\operatorname{PCD}(3,6)$.

Long-term, low-dose clarithromycin therapy improved

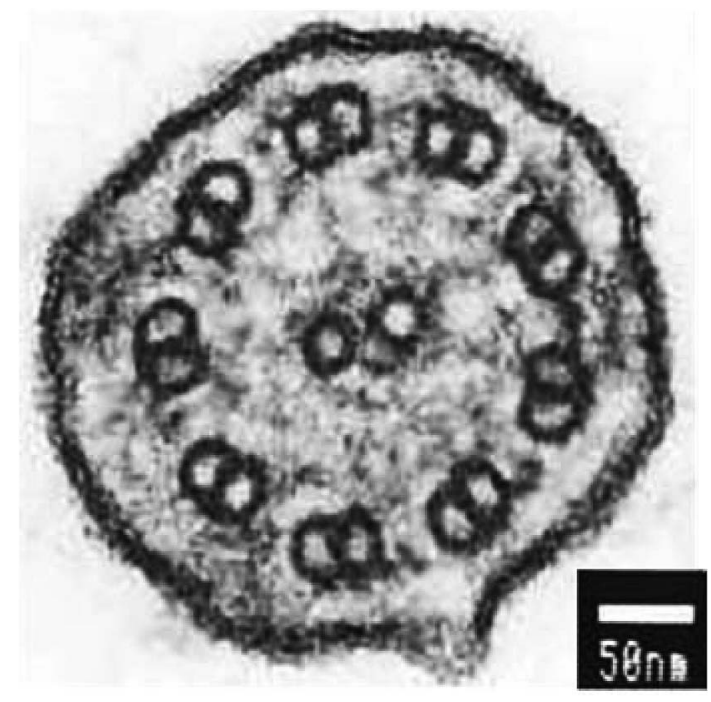

Figure 2. Cross-section of cilia shows absence of inner dynein arms.

productive cough and chest CT findings in the present patient. Macrolide antibiotics including clarithromycin are used as first-line agents to treat acute bacterial infections such as community-acquired pneumonia (7). However, low-dose clarithromycin is generally considered to be below the levels required to inhibit Staphylococcus aureus. In addition, one study in vitro has indicated that clarithromycin does not increase the beat frequency of cilia from rabbit cultured tracheal epithelium (8). Thus, we considered that clarithromycin improves the airway status of PCD patients through modifying the activities of the immune system. The 14membered macrolide, erythromycin, radically improves the clinical outcomes of patients with diffuse panbronchiolitis (DPB) (9), and this report rekindled interest in the use of macrolides as a potential therapy for other inflammatory airway disorders such as $\mathrm{CF}$, bronchiectasis, asthma, obliterative bronchiolitis, chronic obstructive pulmonary disease and chronic rhinosinusitis (10). The 15-membered macrolide azithromycin improves pulmonary function and reduces the rate of respiratory exacerbations in patients with CF (11). The mechanism of these macrolide actions is thought to be due to immune-modifying effects rather than to direct antimicrobial activity (10). Many immune-modifying effects have been reported (12) but we could not clarify which mechanism caused the favorable response in the present patient. Nonetheless clarithromycin decreased airway mucus secretion, inflammatory cytokine and chemokine production and inflammatory cell accumulation, which indicate improved lung involvement.

Homma et al described 8 patients with Kartagener's syndrome (a subset of PCD), in which 7 and 1 were treated with erythromycin and clarithromycin, respectively. However, sputum volume, pulmonary function tests and radiography images were not affected (13). On the other hand, two reports found that three patients with PCD responded to clarithromycin (14) and that one responded to a combination of clenbuterol hydrochloride and azithromycin but not to 


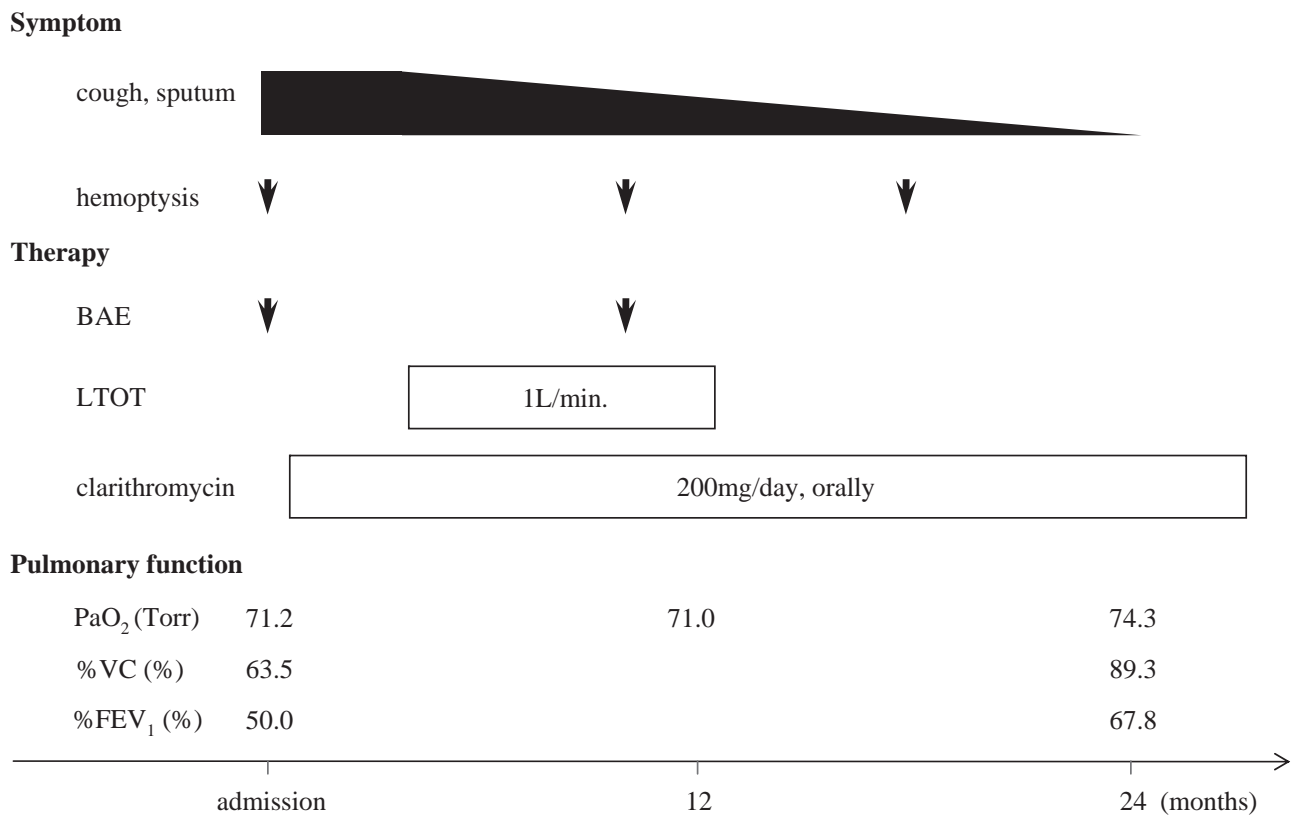

Figure 3. Clinical course. BAE: bronchial artery embolization, LTOT: long-term oxygen therapy

clarithromycin (15). Although the reasons for different responses to macrolides remain to be clarified, reports of patients with DPB or related conditions may suggest some possible mechanisms. Some patients with advanced DPB and extensive bronchiectasis or respiratory failure are refractory to macrolide therapy (9), indicating that disease severity might affect response. The present patient did not have severe symptoms or radiological findings, which might have contributed to his favorable response to macrolide therapy. Other reports $(16,17)$ have demonstrated that the clinical features of human T-cell lymphotrophic virus type 1- or rheumatoid arthritis-associated bronchiolitis are similar to those of DPB, suggesting that multiple disease processes induce the same clinical entity as DPB. However, macrolide therapy improves lung function less effectively in patients with these diseases than in those with DPB $(16,17)$. Furthermore, PCD is a genetically heterogeneous disorder (18), which might also explain the variation in responses to macrolide therapy among PCD patients.

In conclusion, we describe a patient with PCD pulmonary disease that was successfully treated with long-term and low-dose clarithromycin. Further studies are warranted since very few reports have described the effectiveness of this treatment strategy in PCD patients.

\section{Acknowledgement}

We thank Dr. T. Suematsu (Department of Electron Microscopy, Nagasaki University School of Medicine) for analysis of the electron micrographs.

\section{References}

1. Eliasson R, Mossberg B, Camner P, Afzelius BA. The immotilecilia syndrome. A congenital ciliary abnormality as an etiologic factor in chronic airway infections and male sterility. N Engl J Med 297: 1-6, 1977.

2. Bush A, Cole P, Hariri M, et al. Primary ciliary dyskinesia: diagnosis and standards of care. Eur Respir J 12: 982-988, 1998.

3. Bush A, Chodhari R, Collins N, et al. Primary ciliary dyskinesia: current state of the art. Arch Dis Child 92: 1136-1140, 2007.

4. Morillas HN, Zariwala M, Knowles MR. Genetic causes of bronchiectasis: primary ciliary dyskinesia. Respiration 74: 252-263, 2007.

5. Noone PG, Leigh MW, Sannuti A, et al. Primary ciliary dyskinesia: diagnostic and phenotypic features. Am J Respir Crit Care Med 169: 459-467, 2004.

6. Amirav I, Cohen-Cymberknoh M, Shoseyov D, Kerem E. Primary ciliary dyskinesia: prospects for new therapies, building on the experience in cystic fibrosis. Paediatr Respir Rev 10: 58-62, 2009.

7. Mandell LA, Wunderink RG, Anzueto A, et al. Infectious Diseases Society of America/American Thoracic Society consensus guidelines on the management of community-acquired pneumonia in adults. Clin Infect Dis 44 (Suppl 2): S27-S72, 2007.

8. Takeyama K, Tamaoki J, Chiyotani A, Tagaya E, Konno K. Effect of macrolide antibiotics on ciliary motility in rabbit airway epithelium in-vitro. J Pharm Pharmacol 45: 756-758, 1993.

9. Kudoh S, Uetake T, Hagiwara K, et al. Clinical effects of lowdose long-term erythromycin chemotherapy on diffuse panbronchiolitis. Nihon Kyobu Shikkan Gakkai Zasshi 25: 632-642, 1987 (in Japanese).

10. Crosbie PA, Woodhead MA. Long-term macrolide therapy in chronic inflammatory airway diseases. Eur Respir J 33: 171-181, 2009.

11. Wolter J, Seeney S, Bell S, Bowler S, Masel P, McCormack J. Effect of long term treatment with azithromycin on disease parameters in cystic fibrosis: a randomised trial. Thorax 57: 212-216, 2002.

12. Shinkai M, Henke MO, Rubin BK. Macrolide antibiotics as immunomodulatory medications: proposed mechanisms of action. Pharmacol Ther 117: 393-405, 2008.

13. Homma S, Kawabata M, Kishi $K$, et al. Bronchiolitis in Kartagener's syndrome. Eur Respir J 14: 1332-1339, 1999. 
14. Ishiguro $\mathrm{T}$, Takayanagi $\mathrm{N}$, Hijikata N, et al. Primary ciliary dyskinesia. A case report and comparison with 4 previous cases. Nihon Kokyuki Gakkai Zasshi 47: 242-248, 2009 (in Japanese).

15. Itoh $M$, Kishi $K$, Nakamura $H$, et al. A case of immotiledyskinetic cilia syndrome responding to clenbuterol hydrochloride and azithromycin. Nihon Kokyuki Gakkai Zasshi 40: 617-621, 2002 (in Japanese).

16. Hayakawa H, Sato A, Imokawa $S$, et al. Diffuse panbronchiolitis and rheumatoid arthritis-associated bronchiolar disease: similarities and differences. Intern Med 37: 504-508, 1998.

17. Kadota J, Mukae H, Fujii T, Seki M, Tomono K, Kohno S. Clinical similarities and differences between human T-cell lymphotropic virus type 1-associated bronchiolitis and diffuse panbronchiolitis. Chest 125: 1239-1247, 2004.

18. Leigh MW, Pittman JE, Carson JL, et al. Clinical and genetic aspects of primary ciliary dyskinesia/Kartagener syndrome. Genet Med 11: 473-487, 2009.

(C) 2010 The Japanese Society of Internal Medicine http://www.naika.or.jp/imindex.html 